\title{
Heliotropic dust rings for Earth climate engineering
}

\author{
R. Bewick*, C. Lücking, C. Colombo, J.P. Sanchez, C.R. McInnes \\ Advanced Space Concepts Laboratory, University of Strathclyde, Glasgow G1 1XJ, United Kingdom
}

Received 18 June 2012; received in revised form 20 October 2012; accepted 31 October 2012

Available online 7 November 2012

\begin{abstract}
This paper examines the concept of a Sun-pointing elliptical Earth ring comprised of dust grains to offset global warming. A new family of non-Keplerian periodic orbits, under the effects of solar radiation pressure and the Earth's $J_{2}$ oblateness perturbation, is used to increase the lifetime of the passive cloud of particles and, thus, increase the efficiency of this geoengineering strategy. An analytical model is used to predict the orbit evolution of the dust ring due to solar-radiation pressure and the $J_{2}$ effect. The attenuation of the solar radiation can then be calculated from the ring model. In comparison to circular orbits, eccentric orbits yield a more stable environment for small grain sizes and therefore achieve higher efficiencies when the orbit decay of the material is considered. Moreover, the novel orbital dynamics experienced by high area-to-mass ratio objects, influenced by solar radiation pressure and the $J_{2}$ effect, ensure the ring will maintain a permanent heliotropic shape, with dust spending the largest portion of time on the Sun facing side of the orbit. It is envisaged that small dust grains can be released from a circular generator orbit with an initial impulse to enter an eccentric orbit with Sunfacing apogee. Finally, a lowest estimate of $1 \times 10^{12} \mathrm{~kg}$ of material is computed as the total mass required to offset the effects of global warming.
\end{abstract}

(C) 2012 COSPAR. Published by Elsevier Ltd. All rights reserved.

Keywords: Geoengineering; Earth ring; Solar radiation pressure; Earth oblateness

\section{Introduction}

The current consensus within the scientific community is that climate change is likely to have a number of regressive impacts over the coming century. Projections made using climate models over recent years have suggested that the mean global temperature is likely to increase by $1.1^{\circ} \mathrm{C}$ to $6.4{ }^{\circ} \mathrm{C}$ by the end of this century (IPCC et al., 2007). With the continuing industrialisation of the developing world, and the lack of a legally binding international protocol on the tackling of global greenhouse gas emissions, this temperature increase seems likely. While the focus of international efforts should remain with attempts to avoid climate change by reductions of greenhouse gas emissions, it is prudent to investigate methods to mitigate its effects.

\footnotetext{
* Corresponding author.

E-mail address: russell.bewick@strath.ac.uk (R. Bewick).
}

This can be achieved by the deliberate management of the Earth's climate, commonly referred to as climate engineering or geoengineering.

Several proposals for possible geoengineering measures have been made and can generally be placed in two categories; solar radiation management and carbon sequestration (Shepherd et al., 2009). Solar radiation management focuses on the reduction of the flux of sunlight being absorbed by the Earth's atmosphere, by either increasing the Earth's albedo, for example through using reflective roofing materials, or by reducing the flux of sunlight reaching the surface, for example by placing scattering aerosol particles into the stratosphere. Space-based geoengineering (SBGE) fits into the latter category. Alternatively carbon capture techniques aim to deal with the fundamental cause of global warming, an excess of greenhouse gases in the atmosphere, either by direct or indirect methods. Direct methods include capturing $\mathrm{CO}_{2}$ from the air and placing it into storage, whilst an indirect method is the fertilisation 
of the ocean to stimulate increased algal growth, with these algae then leading to increased $\mathrm{CO}_{2}$ uptake.

A report into geoengineering conducted by the Royal Society in 2009 Shepherd et al. (2009) examines the feasibility of all types of schemes based on the criteria of effectiveness, affordability, timeliness and safety. In general the report appears to show that there is no perfect solution, with the schemes that appear most effective suffering in other criteria such as affordability. One of the most effective solutions suggested is the use of space-based solar reflectors to reduce incident solar insolation. Whilst this technique does not appear to be affordable or timely, it does have a key advantage over other schemes; neither the Earth's surface nor atmosphere needs to be physically changed. This is a significant benefit as it reduces the grounds for ethical objections based on the uncertain nature of many geoengineering measures. As an example, the injection of $\mathrm{SO}_{2}$ particles into the stratosphere is rated as having low safety in the Royal Society report. This is because there are indications, from observations of volcanic eruptions, that an increased sulphate concentration in the stratosphere could have adverse effects on the hydrological cycle and ozone layers (Shepherd et al., 2009). Space-based geoengineering will avoid the risks associated with these types of schemes, though there may yet be side effects that cannot be predicted.

It has been estimated that in order to offset the effects of global warming caused by a doubling of $\mathrm{CO}_{2}$ concentration (compared to pre-industrial levels and corresponding to an increase in global temperature of approximately $2{ }^{\circ} \mathrm{C}$ ) solar insolation must be reduced by $1.7 \%$ (Govindasamy and Caldeira, 2000). Similarly for a quadrupling of $\mathrm{CO}_{2}$ the required insolation change is $3.6 \%$ (Govindasamy et al., 2003).

There have been several different proposals to date regarding the reduction of solar insolation using spacebased methods, the key characteristics of which can be seen in Table 1. The methods either utilise a cloud of dust (Bewick et al., 2010; Pearson et al., 2006; Struck, 2007) or solid reflectors or refractors (Angel, 2006; McInnes, 2010; Pearson et al., 2006; Seifritz, 1989) to reduce the level of solar insolation. Typically the methods that require the least mass are those that use solid reflectors/refractors whilst the mass for dust cloud methods are orders of mag- nitude higher. This is mostly due to the increased level of control that can be placed upon the solid devices, hence they can be stationed in optimum positions. Dust clouds cannot be controlled and can only be placed with suitable initial conditions, with subsequent replenishment necessary due to the orbital decay or perturbation of the particle orbits. Conflicting with this, though, is the consideration of the engineering complexity of the system. Whilst dust clouds are a relatively crude method, the material can be readily produced with little processing, whereas solid reflectors must either be manufactured terrestrially, and then launched into position, or manufactured in-situ. Clearly taking this into account, the low rating for affordability and timeliness indicated in the Royal Society report, which only considered solid reflector/refractor devices, can be understood.

A key factor that affects the relative mass of the different methods is the amount of time that the reflectors or dust spends along the Sun-Earth line. For example the method proposed in Struck (2007) to place clouds of dust at the $\mathrm{L}_{4} /$ $\mathrm{L}_{5}$ Lagrange points of the Earth-Moon system has a clear benefit as these points are passively stable. However, as these points effectively orbit around the Earth they are only occasionally in a position to reduce solar insolation. Furthermore, the movement of the clouds will create a flickering effect. On most occasions there will be no change in insolation whilst at those times when the cloud is present the insolation change required will be much greater than the net $1.7 \%$ reduction.

The work presented in this paper aims to investigate the feasibility of using a ring around the Earth in the equatorial plane, as first suggested in Pearson et al. (2006). The difference between the approach presented in Pearson et al. (2006) and this paper is that novel families of orbits are proposed through consideration of the orbital mechanics of the high area-to-mass ratio dust grains, including solar radiation pressure (SRP) and the effect of the Earth's oblateness, the $J_{2}$ effect. It will be shown that SRP has a significant effect on the orbital evolution of small dust particles. These particles are more mass efficient at intercepting solar photons, i.e. they have a higher area-to-mass ratio, but at the same time they are also more prone to be affected by perturbative forces, and thus more likely to de-orbit. Grains with the sizes and at the altitudes suggested in

Table 1

Key properties of the different geoengineering methods proposed in literature.

\begin{tabular}{|c|c|c|c|c|}
\hline Position & Method & Insolation change $[\%]$ & Required mass [kg] & Refs. \\
\hline Earth orbit & Dust ring & 1.6 & $2.3 \times 10^{12}$ & Pearson et al. (2006) \\
\hline Earth orbit & Dust ring & 1.7 & $2.0 \times 10^{12}$ & This paper \\
\hline Earth orbit & Solar reflector & 1.6 & $5.0 \times 10^{9}$ & Pearson et al. (2006) \\
\hline Earth-Moon $\mathrm{L}_{4} / \mathrm{L}_{5}$ & Dust Cloud & 1.4 & $2.1 \times 10^{14}$ & Struck (2007) \\
\hline Sun-Earth $\mathrm{L}_{1}$ & Solar Reflector & 1.8 & $2.6 \times 10^{11}$ & McInnes (2010) \\
\hline Sun-Earth $\mathrm{L}_{1}$ & Solar Refractor & 1.8 & $2.0 \times 10^{10}$ & Angel (2006) \\
\hline Sun-Earth $\mathrm{L}_{1}$ & Dust Cloud & 1.7 & $1.9 \times 10^{10}$ & Bewick et al. (2012) \\
\hline
\end{tabular}


Pearson et al. (2006) will de-orbit rapidly as will be shown in a later section. A balance between mass efficiency and orbital stability must therefore be found.

\section{Orbital dynamics}

In this section the orbital dynamics of Earth orbiting dust is discussed. The in-plane orbital evolution due to solar radiation pressure and the $J_{2}$ effect is analysed analytically. The out-of-plane effects and resulting evolution of the inclination are investigated numerically.

\subsection{Hamiltonian model of dust orbital dynamics}

In order to tackle the problem analytically, a simplified planar model is used. We describe the dynamics of dust in an Earth-centred synodic reference frame. We consider a dust grain orbiting on a planar orbit (i.e. the orbit has zero inclination) with the simplifying assumption that the equatorial plane is in the ecliptic (i.e. the obliquity angle of the ecliptic with respect to the equator is set to zero). As will be shown later, this simplifying assumption provides an adequate description of the evolution of the dust with the level of accuracy intended for the paper, while allowing an analytical description of the underlying dynamics of the dust ring.

In the frame as described above, three parameters are needed to define any in-plane orbit. The eccentricity, $e$, semi-major axis, $a$, and the angle between the direction of the solar radiation and the radius of the perigee with respect to the Earth, $\phi$. The position of the dust grain is also defined with respect to the direction of the sunlight using the angle $\theta$, where $\theta=\phi+f$ with true anomaly $f$. Fig. 1 shows the geometry of the reference frame.

Work presented by Hamilton and Krivov (1996) and Krivov and Getino (1997) on the orbital dynamics of high area-to-mass ratio spacecraft introduces an approximate Hamiltonian for planar orbits under the effects of solar radiation pressure and the Earth's oblateness (Krivov

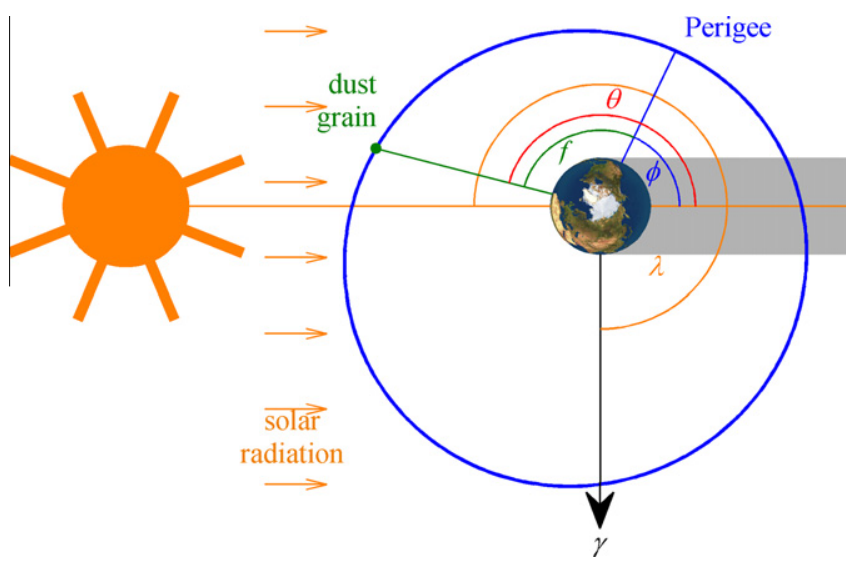

Fig. 1. Geometry of an in-plane orbit in the synodic reference frame. and Getino, 1997). This analytical model does not consider eclipses and the tilt of the Earth's rotational axis with respect to the ecliptic plane. As a consequence, the semimajor axis remains constant and the evolution of the orbit can be described only by its eccentricity $e$, defining the changing shape of the orbit, and solar radiation perigee angle $\phi$, defining the orientation. The change of orbital elements due to solar radiation pressure and $J_{2}$ with respect to the progression of the angle between the position of the Sun on the ecliptic with respect to the vernal equinox, $\lambda_{\odot}$, is (Krivov and Getino, 1997):

$\frac{d e}{d \lambda_{\odot}}=-\alpha \sqrt{1-e^{2}} \sin \phi$

$\frac{d \phi}{d \lambda_{\odot}}=-\alpha \frac{\sqrt{1-e^{2}}}{e} \cos \phi+\kappa \frac{1}{\left(1-e^{2}\right)^{2}}-1$

where $\alpha$ is the radiation pressure parameter and $\kappa$ the $J_{2}$ effect parameter, defined by:

$\begin{aligned} \alpha & =\frac{3}{2} a_{S R P} \frac{a^{2}}{\mu} \frac{n}{n_{\odot}} \\ \kappa & =\frac{3}{2} J_{2} \frac{R_{E}^{2}}{a^{2}} \frac{n}{n_{\odot}}\end{aligned}$

where $n=\sqrt{\mu / a^{3}}$ is the orbit angular velocity of the dust grain on its Earth-centred orbit, $\mu$ is the gravitational parameter of the Earth, $J_{2}$ its second order zonal harmonic coefficient, $R_{E}$ its radius and $n_{\odot}$ the orbit rate of the Earth around the Sun. While $\kappa$ is only a function of the semi-major axis, $\alpha$ is also dependent on $a_{S R P}$, the acceleration due to solar radiation pressure (SRP). For circular objects with radius $r$ and density $\delta$ it can be calculated as follows (Krivov et al., 1995):

$a_{S R P}=\frac{F_{\odot}}{c} \frac{4}{3 \delta r}$

where $F_{\odot}$ is the solar flux and $c$ is the speed of light.

The resulting Hamiltonian is (Krivov and Getino, 1997):

$H=-\sqrt{1-e^{2}}+\alpha e \cos \phi-\frac{\kappa}{3{\sqrt{1-e^{2}}}^{3}}$

For a certain range of semi-major axis and area-tomass-ratios, the Hamiltonian in Eq. (6) allows, among all its stationary points, a stable equilibrium (i.e., $d e / d \lambda_{\odot}=0$ and $\left.d \phi / d \lambda_{\odot}=0\right)$, at $\phi=0$ with eccentricity $e_{0}$ as shown by Colombo et al. (2012). This equilibrium point represents frozen orbits with their apogee pointing in the direction of the Sun. These orbits are therefore termed heliotropic orbits. If the solar radiation pressure parameter is zero, $\alpha=0$ (i.e. without the effect of SRP), the equilibrium at $e_{0, J_{2}}$ corresponds to an orbit with a frozen orientation with respect to the Sun solely due to the $J_{2}$ effect. Such an orbit would have its apse-line precessing at the same rate as the Earth's motion around the Sun. While the SRP parameter is zero, the equilibrium exists for any orbit orientation. However, as soon as the SRP parameter is non-zero the equilibrium can only be found for 


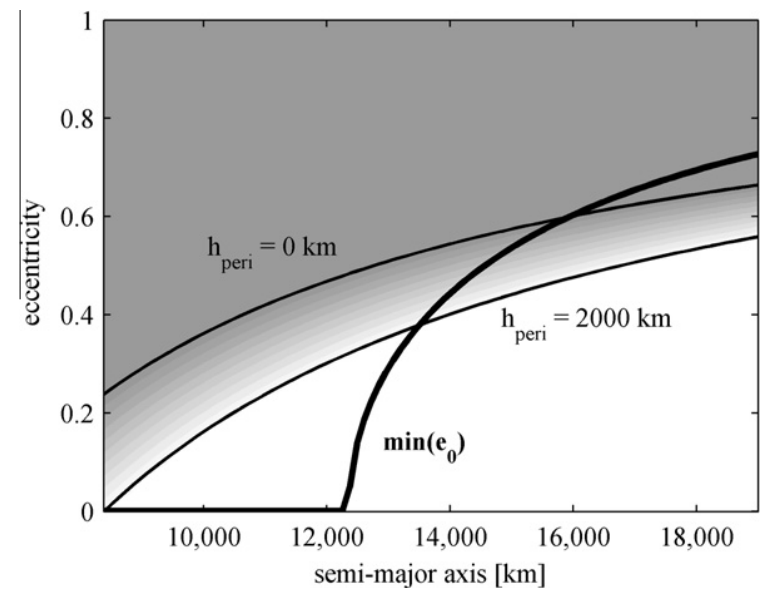

Fig. 2. Minimum eccentricity $(\alpha=0)$ of the heliotropic equilibrium orbit $\min \left(e_{0}\right)$ as a function of semi-major axis. The gradient in the background corresponds to perigee altitudes of the corresponding orbit within $h_{\text {peri }}=0 \mathrm{~km}$ and $h_{\text {peri }}=2000 \mathrm{~km}$, specifically marked, where the effect of drag is non-negligible.

Sun-pointing apogees. For increasing values of the SRP parameter the equilibrium eccentricity $e_{0}$ increases, with the $J_{2}$-only equilibrium eccentricity $e_{0 . J_{2}}$ (i.e., $\alpha=0$ ) being the minimum boundary value at a given semi-major axis (Colombo et al., 2012). Fig. 2 shows $e_{0, J_{2}}=\min \left(e_{0}\right)$ as a function of semi-major axis. In the background a gradient shows the perigee altitude within a range of $h_{\text {peri }}=0 \mathrm{~km}$ and $h_{\text {peri }}=2000 \mathrm{~km}$ specifically marked. $2000 \mathrm{~km}$ is approximately the altitude from which the effect of drag on the orbital evolution is negligible, even for very high area-to-mass ratios (Colombo and McInnes, 2011). A ring of dust at or beyond this distance from the Earth will remain in place infinitely. It can be seen from the figure that heliotropic orbits do not exist above c. $16,000 \mathrm{~km}$ semimajor axis. Above c. $13,500 \mathrm{~km}$ semi-major axis they become unstable due to drag.

\subsection{In-plane orbital evolution of dust}

A small grain size is desirable as the mass efficiency of the geoengineering measure is inversely proportional to grain radius. If a grain with a specific area-to-mass ratio is released in any orbit, its orbit will then evolve by librating or rotating around its equilibrium eccentricity (Colombo et al., 2012). The greater the initial distance from the libration point in the phase space the larger the maximum eccentricity reachable. Therefore, it is more efficient to release the dust grains (with different area-to-mass ratios) at a higher initial eccentricity to prevent them from de-orbiting due to drag. Fig. 3 compares the orbital evolution of grains with different radii when they are released in a circular and elliptical orbit with the same semi-major axis. A grey colour marks the area in which the orbits will experience drag and we consider grains which pass through this area as "lost". It can be seen that while for an initially circular orbit all grains with a radius smaller than 13 (a) Released in circular orbit

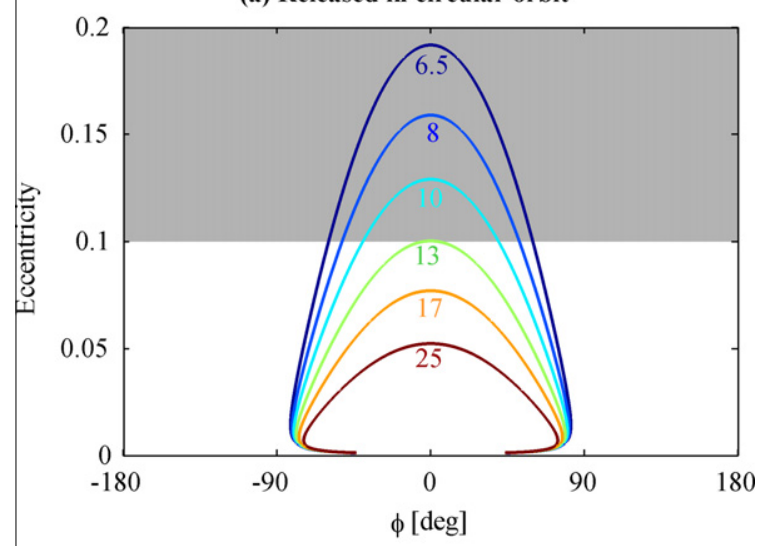

(b) Released in eccentric orbit

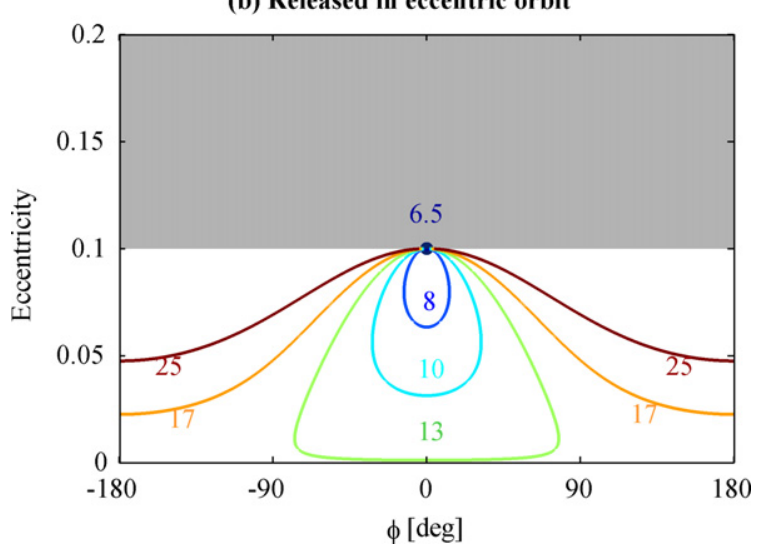

Fig. 3. Orbital evolution in the $e-\phi$ phase space for grains with different radii from 6.5 to 25 microns released at c. $9300 \mathrm{~km}$ semi-major axis with $e=0$ (a) and $e=e_{\text {crit }}$ (b). The grey zone marks the area in which the perigee of the orbit is closer than $2000 \mathrm{~km}$ to the surface of the Earth.

microns will be lost (see Fig. 3(a)), in the elliptical case grains as small as 6.5 microns survive (see Fig. 3(b)). It follows that release at the critical eccentricity yields the best results and that the smallest possible grain radius at any semi-major axis is the one which has its equilibrium point at the critical eccentricity.

\subsection{Choice of feeder orbit}

We can determine the smallest possible grain radius as a function of semi-major axis by finding the particle radius for which the equilibrium eccentricity $e_{0}$ is equal to $e_{\text {crit }}$, the critical eccentricity corresponding to the smallest allowable perigee height $h_{\text {peri }}$ :

$e_{\text {crit }}=1-\frac{R_{E}+\min \left(h_{\text {peri }}\right)}{a}$

To find the minimum particle radius we set $\phi=0$ in Eq. (2), set $d \phi / d \lambda_{\odot}$ equal to zero, substitute $e$ with $e_{\text {crit }}$ in Eq. (7), solve for $\alpha$ and combine with Eqs. (3) and (6) to get:

$r_{\min }=\frac{2 F_{\odot}}{c n_{\odot} \delta} \sqrt{\frac{a}{\mu}} \frac{{\sqrt{1-e_{c r i t}^{2}}}^{5}}{e_{c r i t}\left(\kappa(a)-\left(1-e_{c r i t}^{2}\right)^{2}\right)}$ 


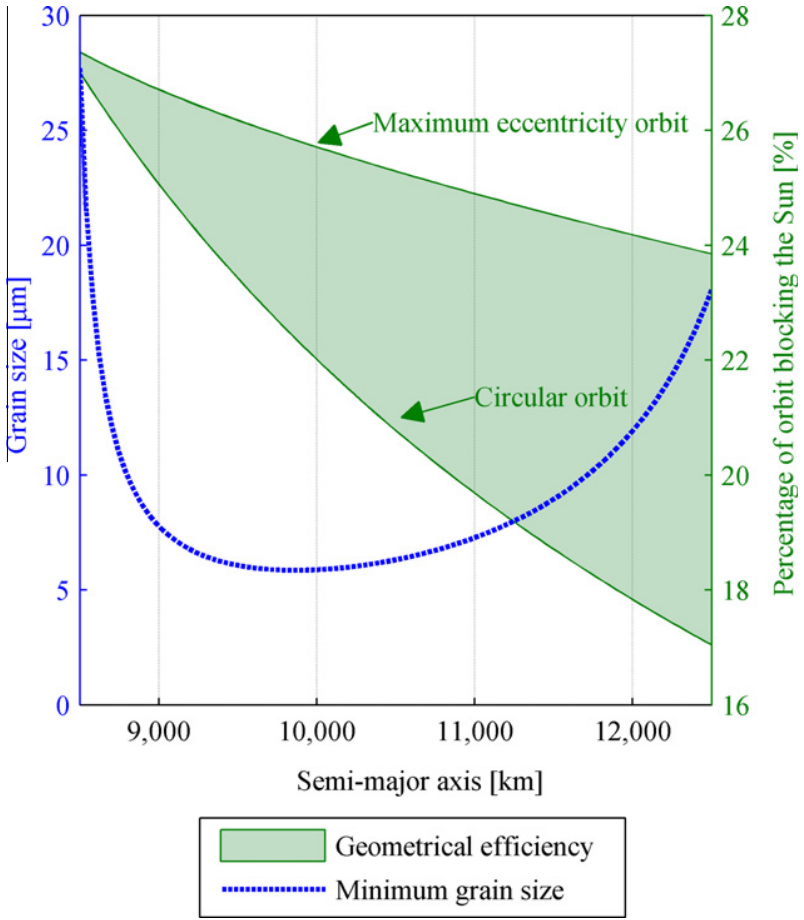

Fig. 4. Geoengineering efficiency indicators (grain size and orbit geometry) with semi-major axis. The minimum grain size which can be deployed at the given semi-major axis without rapid decay is given on the left axis. The geometrical efficiency is given on the right axis as a range from a circular orbit to an orbit with critical eccentricity and Sun-pointing apogee.

The density $\delta$ for the asteroid grains used is $3500 \mathrm{~kg} \mathrm{~m}^{-3}$ as in Wilck and Mann (1996).

Another measure of the efficiency of a ring for geoengineering is how much of its orbital period a grain spends blocking solar radiation. This is dependent on the orbital geometry and takes into account that a grain travels slower at apogee than at perigee. We term the resulting percentage (of time spent in the useful region) the geometrical efficiency which can be found numerically.

Using these indicators of efficiency we can next choose the semi-major axis likely to be most efficient for geoengineering. Fig. 4 shows the dust grain minimum radius and the geometrical efficiency of a circular orbit and an orbit with $e=e_{\text {crit }}$ as a function of semi-major axis. The minimum grain size is important as smaller grains have higher area-to-mass ratios and thus offer better mass efficiency when used to block sunlight. For the geometrical efficiency both the circular and elliptical orbits are considered because we will be releasing grains with a distribution of radii and not all of these have an equilibrium at the critical eccentricity. Thus, some grains will librate in eccentricity between $e \geqslant 0$ and $e=e_{0}$. A good compromise between low minimum grain size and high geometrical efficiency is desired. Considering the results in Fig. 4, an advantageous initial orbit for the dust ring, referred thereafter as the feeder orbit, has a Sun-pointing apogee with radius $10,250 \mathrm{~km}$ $(a=9318 \mathrm{~km})$ and an eccentricity of $e_{f}=0.1$.

\section{4. $3 D$ orbital evolution of dust}

In the following section the simplified assumption used in Section 2.1 of a zero obliquity angle of the ecliptic with respect to the equator will be removed. The actual evolution of dust particles differs from the one predicted by the Hamiltonian equation (6) because of the 23.5 degree tilt angle of the equator with respect to the ecliptic plane. As a consequence, a dust ring initially positioned on the ecliptic plane will be subject to a nodal drift, due to the effect of $J_{2}$ and SRP and out of plane oscillations under solar radiation pressure. As analysed in Colombo et al. (2012), the simplified planar phase space approximation used in Section 2 maintains its structure for small tilt angle (less than $30^{\circ}$ as in the Earth case) and small inclinations. Fig. 5 represents the 3D evolution of the ring over a period of 20 years, propagated through a set of non-singular Lagrangian elements derived by Krivov et al. (1995) and reported in Colombo et al. (2012) to correct a typo in Krivov's paper (in the SRP term). The effect of eclipses is neglected. The harmonic oscillation in the $e-\phi$ phase space is still recognisable, although the single line for a given area-to-mass ratio extends to a band (see Fig. 5(a)). Note that a three-dimensional definition of the solar radiation perigee angle $\phi$ is adopted here as

$\phi=\Omega+\omega-\left(\lambda_{\odot}-\pi\right)$

where $\Omega$ and $\omega$ are respectively the argument of the ascending node (from the vernal equinox) and the argument of
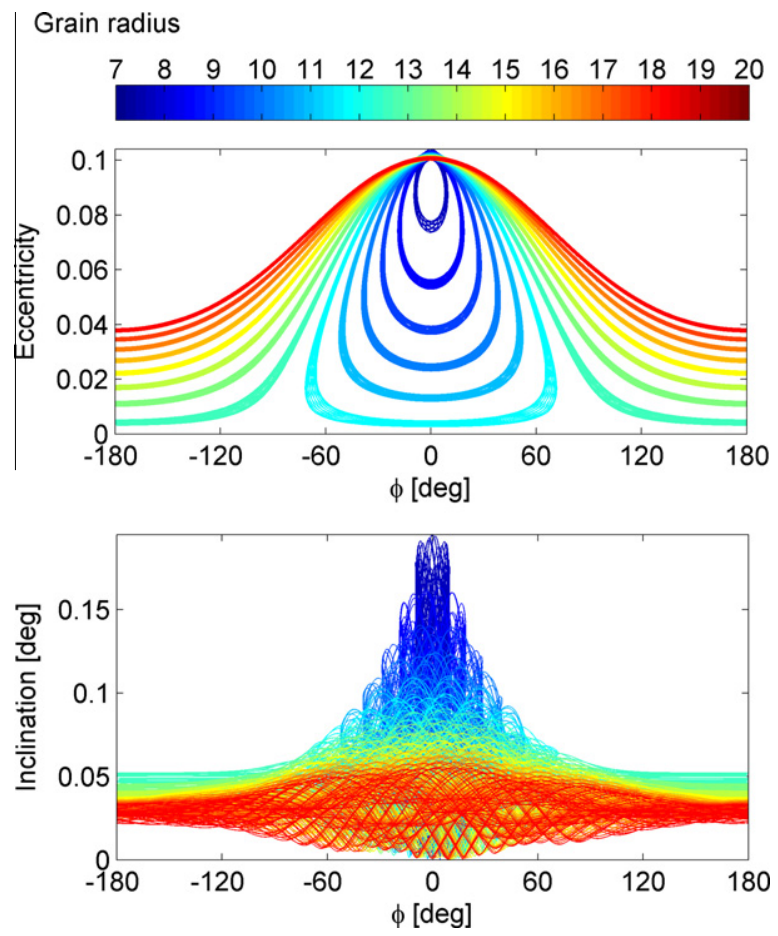

Fig. 5. 3D evolution of the dust ring over a period of 20 years. (top) Planar structure in the $\phi-e$ phase space and (bottom) vertical structure in the $\phi-i$ phase space. 


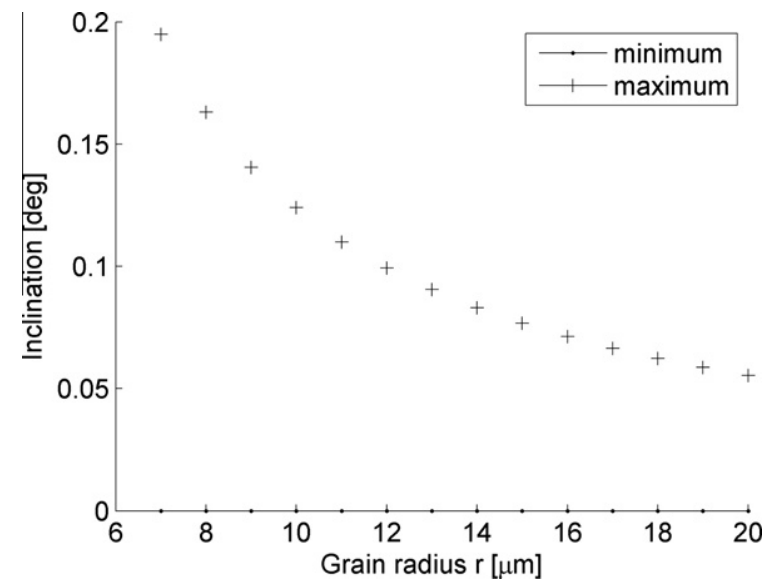

Fig. 6. Maximum (cross) and minimum (dot) inclination change on the equator as function of the grain radius.

perigee of the grain's orbit, and $\lambda_{\odot}$ defines the true longitude of the Sun on the ecliptic.

A full characterisation of the Earth ring evolution is given when we consider also the perturbations to the inclination and the ascending node, which describes the vertical structure (see Fig. 5(b)). The dust grains display complex inclination time history, however the magnitude of the inclination changes are limited to a maximum of $0.2^{\circ}$, this occurring for the smallest grains, as shown in Fig. 6.
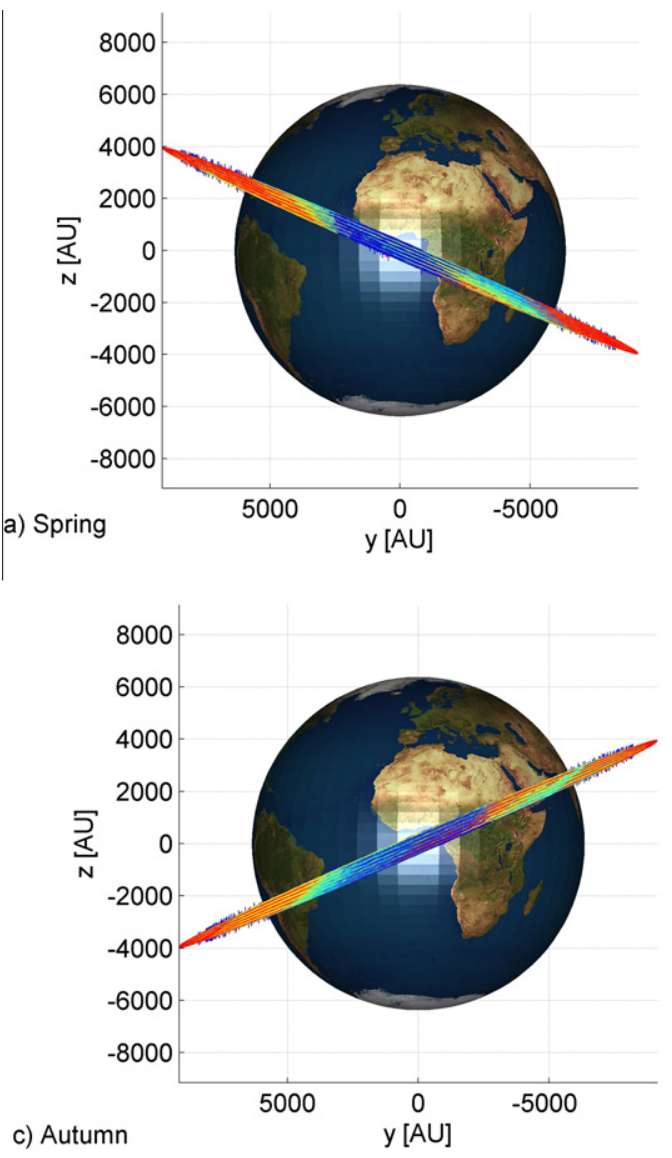

Depending on the season, the apogee point of the heliotropic orbits will oscillate below and above the ecliptic plane, as can be seen in Fig. 7. This figure represents the seasonal orbital evolution of the ring over 20 years. The colour scale represents the range of dust radii used.

\section{Geoengineering scenario}

\subsection{Dust source}

Some consideration must be given to the source of dust that is to be dispersed from the feeder orbit as it is expected that a significant quantity is required. Previous space-based geoengineering concepts have suggested the use of dust sourced from the Earth, Moon, asteroids and comets (Bewick et al., 2012; Pearson et al., 2006; Struck, 2007). It has been shown that the amount of asteroid material that can be captured into a weakly bound Earth orbit with a threshold velocity lower than that of lunar escape velocity, $2.37 \mathrm{~km} \mathrm{~s}^{-1}$, is $6 \times 10^{13} \mathrm{~kg}$ (Sanchez and McInnes, 2011). For the material required in this SBGE scenario an extra velocity increment of $2.7 \mathrm{~km} \mathrm{~s}^{-1}$ would be required to lower the orbit to the position of the feeder orbit, therefore doubling the velocity requirement.

Despite the additional costs of transporting asteroid material to a medium Earth orbit, the use of captured
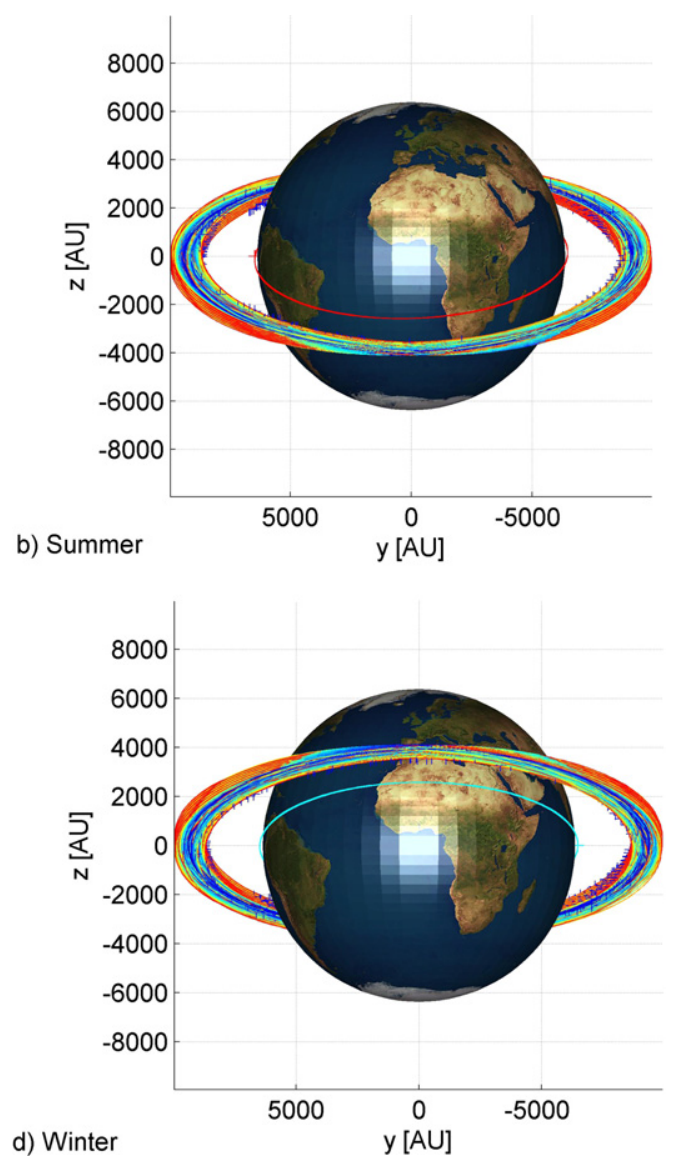

Fig. 7. Ring seasonal evolution over 20 years. 
asteroid resources would still be more efficient than lifting material from the surface of the Earth. This also applies for schemes that require solid reflectors to block solar radiation where large devices must be manufactured and then launched into the correct position. Lifting such large masses (of the order of $10^{10} \mathrm{~kg}$ ) is beyond current launch capabilities. Using asteroid material rather than lunar dust has the added benefit that the material can be sourced and ground to finer grain sizes, if required, whilst in the feeder orbit before being released, thus removing the requirement to transport material from the Moon.

An additional source of material could possibly be spent rocket stages and other debris that can be ground to dust. The NORAD database of tracked space objects reveals that there are over 1000 spent rocket stages and over 10,000 pieces of other debris in LEO and MEO orbits. Assuming a minimum mass of 1 metric tonne per rocket stage gives a lower bound of $1 \times 10^{6} \mathrm{~kg}$ in this region. This is a relatively small amount of material in comparison to the scale of typical geoengineering schemes and, given that the orbits of these debris objects will be widely dispersed, this is not a viable option for populating the dust ring.

The asteroid is envisaged to be captured into a circular, equatorial generator orbit with semi-major axis $a_{g}=$ $10,250 \mathrm{~km}$ (see Fig. 8). It follows that $10,250 \mathrm{~km}$ is also the radius of the apogee for the feeder orbit selected in the previous section. Since the area-to-mass ratio of an asteroid is clearly small, the effect of solar radiation pressure is negligible. The orbit is thus assumed to be unperturbed.

The dust grains will be continuously extracted from the asteroid, milled to achieve a certain radii distribution and collected during one orbit. Whenever the generator passes $\theta=\pi$ (we consider here as conventional direction for measuring the angle $\theta$ on a circular orbit the positive $x$-axis) it ejects the collected dust with the correct $\Delta v$ to inject it into

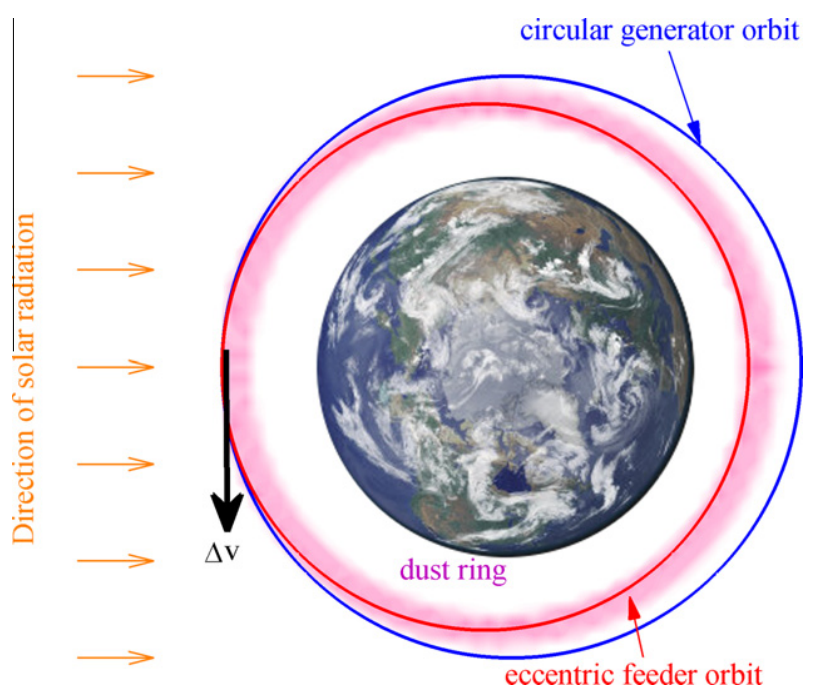

Fig. 8. Illustration of the mission concept with generator orbit, feeder orbit and the resulting dust ring.
Table 2

Values for mean and standard deviation of the three dust grain distributions.

\begin{tabular}{lll}
\hline Distribution & Mean $\mu$ & Standard deviation $\sigma$ \\
\hline D1 & -11.5 & 0.1 \\
D2 & -11.35 & 0.15 \\
D3 & -11.2 & 0.25 \\
\hline
\end{tabular}

the feeder orbit using a mass driver. From this feeder orbit the grains will then evolve and spread due to their different area-to-mass ratios and thus form the dust ring. Because the period of the generator orbit and the feeder orbit are different, grains will be distributed at all positions in the orbit. After a maximum of one year all positions on the libration curves in the phase space will be filled and after 4 years the orbits will be spread over all inclinations smaller than $0.2^{\circ}$ with respect to the equator. Fig. 8 shows a scale illustration of the concept.

\subsection{Dust grain size distributions}

The milled dust is assumed to be distributed log-normally in grain radius $r$ :

$f_{\mu, \sigma}(r)=\frac{1}{r \sigma \sqrt{2 \pi}} e^{-\frac{(\ln r-\mu)^{2}}{2 \sigma^{2}}}$

Three possible distributions have been chosen. D1 is an optimistic estimate with small mean and low standard deviation. D2 is a realistic distribution and achievable with existing terrestrial milling machinery. ${ }^{1} \mathrm{D} 3$ is a pessimistic estimate with high mean and large standard deviation. Table 2 shows the chosen values for mean and standard deviation and Fig. 9 shows the three distributions as probability density functions.

\section{Ring model}

To derive the mass needed for an average insolation reduction of $1.7 \%$ over one year the attenuation through the ring must be known. This is achieved by using the Beer-Lambert law;

$I=I_{0} e^{-\Lambda l}$

where $I_{0}$ is the solar constant before passing through the ring, $\Lambda$ is the attenuation coefficient and $l$ is the path length. The attenuation coefficient at any given point is calculated by;

$\Lambda=\int_{r_{1}}^{r_{2}} \pi r^{2} \rho(r) d r$

where $r$ is the grain radius and $\rho(r)$ is the number density of grains at each radius. Since the attenuation coefficient is

\footnotetext{
${ }^{1}$ http://www.zenithcrusher.com/ Last accessed 05 Sep 2011; http:// www.crushermills.com/ Last accessed 05 Sep 2011.
} 


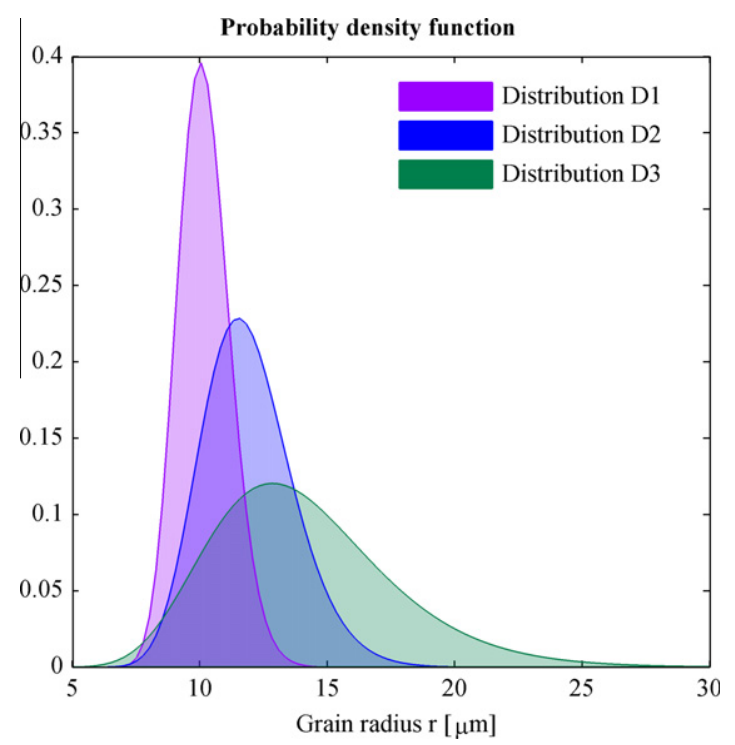

Fig. 9. Probability density functions for the three distributions of grain radii considered.

likely to change through the ring, the attenuation coefficient must be integrated over the path length and hence Eq. (10) becomes;

$I=I_{0} e^{-\int_{0}^{l} \Lambda(l) d l}$

To determine the attenuation coefficient for the different distributions a phase space density model must to be built. To achieve this, a two dimensional model of the ring will be constructed, using the in-plane dynamics discussed before. The attenuation coefficient is then calculated for this inplane evolution of the dust, which, as seen in Section 2.4, reproduces well the dynamical behaviour of the ring. Then the orbital plane is tilted with respect to the ecliptic plane and the inclination change added. Thus, a third dimension is added by approximating the out of plane evolution with a change of plane.

\subsection{In-plane model}

An expression is needed to find the radius of a grain passing through any point $(\phi, e)$ in the phase space. This can be derived by setting the Hamiltonian Eq. (6) with $(\phi$ ,e) equal to the Hamiltonian of the feeder orbit with $\left(0, e_{f}\right)$ and solving for $\alpha$. Then applying Eqs. (3) and (5), gives:

$$
r(\phi, e)=\frac{\frac{6 F_{\odot}}{c n_{\odot} \delta} \sqrt{\frac{a}{\mu}}\left(e_{f}-e \cos \phi\right)}{3\left(\sqrt{1-e_{f}^{2}}-\sqrt{1-e^{2}}\right)+\kappa\left(\frac{1}{\sqrt{1-e_{f}^{2}}}-\frac{1}{{\sqrt{1-e^{2}}}^{3}}\right)}
$$

The probability of finding any grain in a differential element around a given $(\phi, e)$ in the phase space can then be determined. This is achieved by calculating the required radius of the grain using Eq. (13). Then the probability of any grain having the calculated radius can be found with the cumulative probability density function Eqs. (14) and (15). This gives the fraction of all grains whose evolution passes through the considered differential phase space element.

$$
\begin{aligned}
P(r & \left.\in\left[r_{0}-d r, r_{0}+d r\right]\right) \\
& =F_{\mu, \sigma}\left(r_{0}+d r\right)-F_{\mu, \sigma}\left(r_{0}-d r\right)
\end{aligned}
$$

with

$F_{\mu, \sigma}(r)=\frac{1}{\sqrt{2 \pi}} e^{-\frac{(\ln r-\mu)^{2}}{2 \sigma^{2}}}$

Next the specific density at this position is calculated. This is the fraction of grains passing through the considered differential box in the phase space. Combining the specific density at the considered location in the phase space with the probability density and grain size calculated before we will obtain the total fraction of grains in this element of the phase space.

The specific density is found numerically by first calculating the libration period, in the phase space, of a grain of a given size. Then this is compared with the time derivative of the orbital parameters in the phase space at the given position calculated using Eqs. (1) and (2). Fig. 10 shows the resulting number density in the phase space for the three distributions of grain radii introduced in the previous section. It is shown relative to the average number density in the phase space. As expected the highest density can be found in the release position, the feeder orbit, at $\left(0, e_{f}\right)$. The narrowest distribution, D1, shows high density in higher eccentricity orbits with the apogee facing within $45^{\circ}$ of the Sun. The wider the grain size distribution the more dispersed the dust cloud is in the phase space. It can be assumed that the narrowest distribution has the highest proportion of grains blocking sunlight. To ascertain this assumption the dust density around the Earth in polar coordinates is calculated next.

The polar coordinate system chosen is a rotational reference frame with fixed orientation towards the sun. Any position in the orbital plane is determined by $R$, the distance to the centre of the Earth, and $\theta$, the angle with respect to the direction of solar radiation as shown in Fig. 1.

Fig. 11 shows the accumulated in-plane number density as a function of $\theta$ for the three different initial distributions. It can be seen that the narrowest distribution D1 indeed has the largest number of particles facing the sun, about $15 \%$ more than the orbit average.

The in-plane number density, which is the probability of finding a grain in a given area of the orbital plane, can then be used to calculate $\Lambda_{0}$, the in-plane attenuation coefficient, using Eq. (11). The in-plane coefficient differs from the final value to be used as here the number density is in terms of area not volume and hence there is an intermediate step to calculate the true attenuation coefficient. 
(a) Number density $\log _{10}(\rho(\mathrm{D} 1))$

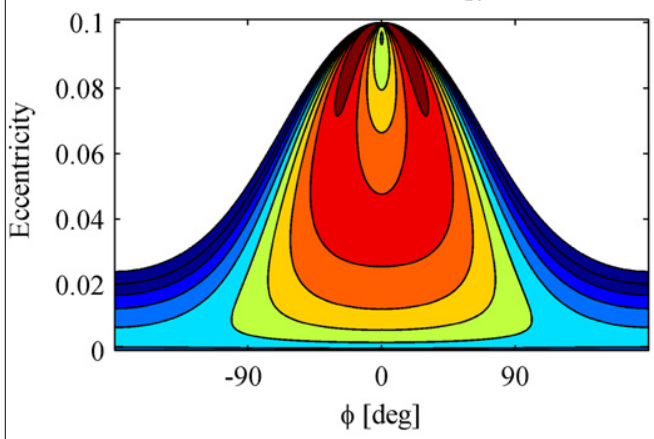

(b) Number density $\log _{10}(\rho(D 2))$

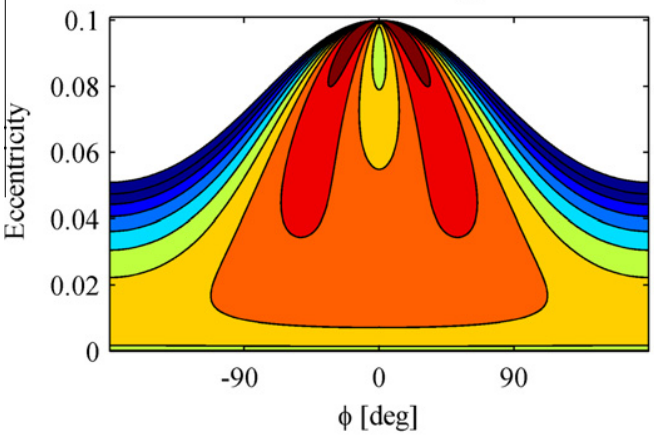

(c) Number density $\log _{10}(\rho(D 3))$

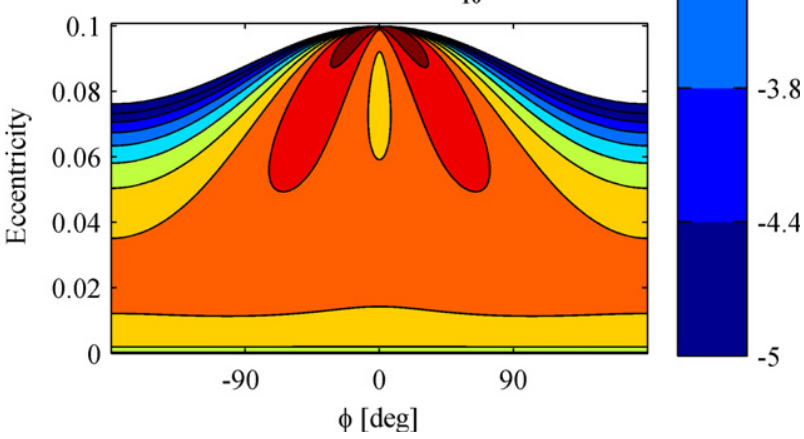

Fig. 10. Number density relative to average number density in the phase space for different grain radius distributions.

Fig. 12 shows the in-plane attenuation coefficient relative to the maximum attenuation for the three different distributions. The maximum attenuation occurs predictably at the intersection with the generator orbit at $\theta=\pi$ and $R=a_{f}\left(1+e_{f}\right)$. The feeder orbit itself can also be seen in all three figures. Apart from the feeder orbit, a wider dust ring is discernible in all three figures with attenuation values of order $10 \%$ of the maximum. The narrowest distribution D1 has the narrowest ring measuring approximately $600 \mathrm{~km}$. The ring of the widest distribution D3 is about twice as wide at approximately $1200 \mathrm{~km}$. Although distribution D1 is favourable because of its small average grain radius and its higher geometrical efficiency, D3 could have an advantage with the wider ring allowing the burden of the reduced insolation to be stretched over a larger area of the Earth, thus making the shadowing effect less pronounced.

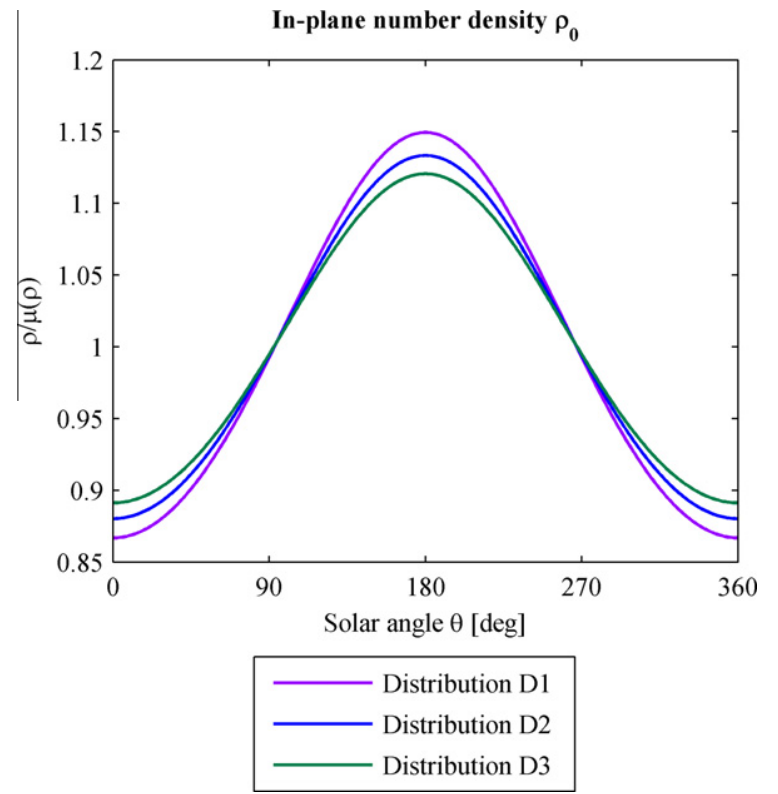

Fig. 11. Sum of the in-plane number density for different solar angles $\theta$ in relation to the average.

\subsection{Three dimensional model}

To fully determine the mass of dust required to offset the $1.7 \%$ insolation change a $3 \mathrm{D}$ model of the ring must be constructed. The first stage in this process is to transfer the in-plane attenuation coefficient into a three dimensional attenuation coefficient. This is estimated by dividing the inplane coefficient by the out of plane width at radius $R$;

$\Lambda(R, \theta)=\frac{\Lambda_{0}(R, \theta)}{2 R \delta i}$

To construct the $3 \mathrm{D}$ model it is assumed that as the distance between the ring and the Earth is small in comparison to the distance to the Sun, the solid angle that it subtends will have a negligible effect on the results of this paper. Therefore the incoming solar flux is assumed to be a plane wave. Hence, the overall attenuation over the Earth's surface shall be determined by integrating along the $x$-axis for given values of $y$ and $z$.

The geometry of the problem can be seen in Fig. 13. For a given inclination of the ring to the Sun-line the exit and entry points of the integration can be calculated and the Beer-Lambert law can be applied between these two positions. For each point along the integration line the position must be transformed from Cartesian coordinates to the $(R, \theta)$ system that describes the attenuation coefficient. This value can then be multiplied by the path length to give the fractional intensity before being integrated.

To find an accurate determination of the mass required, this process must be averaged over time. This is necessary as the angle between the equatorial and ecliptic planes, from the viewpoint of the sun, will change over the course of a year. It can be found that the inclination angle between the Sun-line and the equatorial plane for a circular orbit 
(a) In-plane attenuation coefficient $\Lambda_{0}$ (D1)

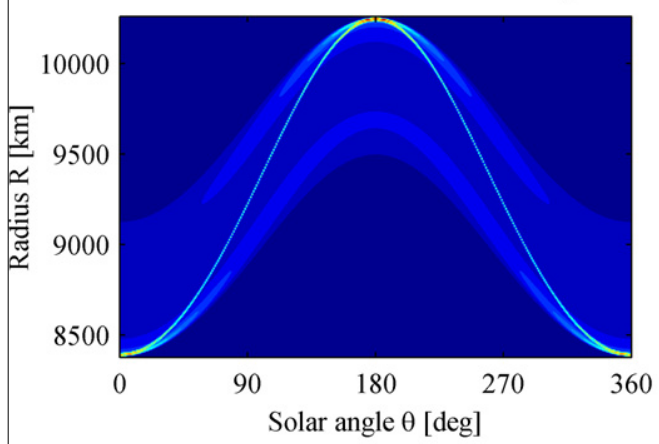

(b) In-plane attenuation coefficient $\Lambda_{0}(\mathrm{D} 2)$

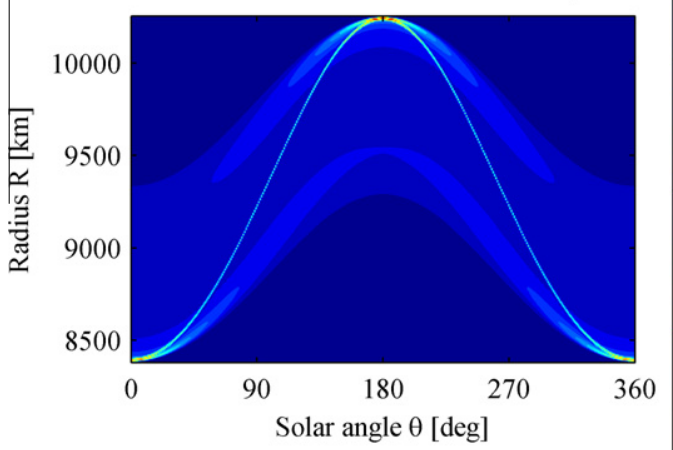

(c) In-plane attenuation coefficient $\Lambda_{0}(\mathrm{D} 3)$

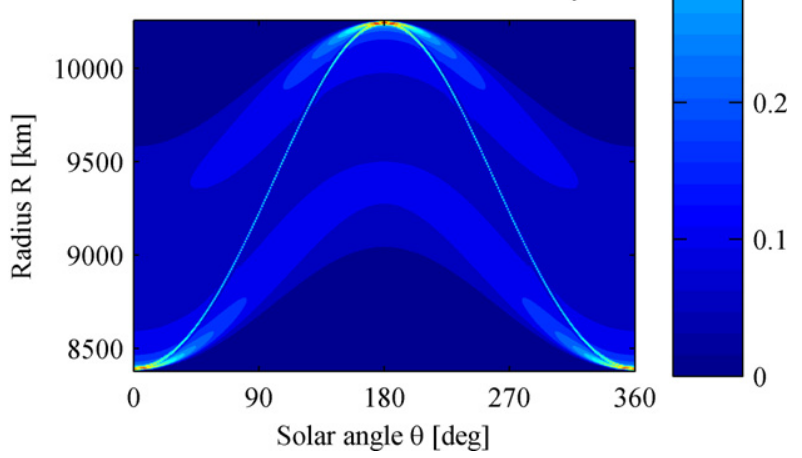

Fig. 12. In-plane attenuation coefficient in polar coordinates and in relation to the maximum attenuation.

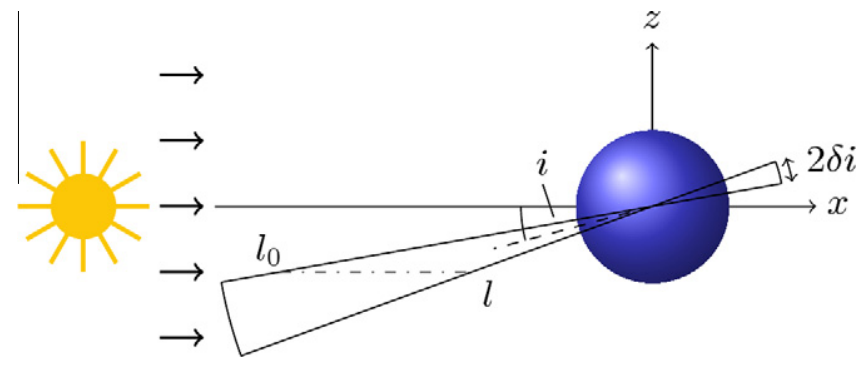

Fig. 13. Diagram of the 3D ring model (northern summer pictured).

around the Sun is described by a sinusoidal oscillation, assuming the starting point is at the northern hemispheres spring. The view of the Earth, as seen from the Sun, is shown in Fig. 7 for different times of the year. Next the mass required for the three different dust distributions can be found.

\section{Results and discussion}

The mass required for each of the dust distributions described previously can be seen in Fig. 14. It can be seen, as would at first be expected, that the distribution with the greatest fraction of its number density along the Sun line requires the lowest mass of $5.94 \times 10^{11} \mathrm{~kg}$. This is additionally due to the smaller grain sizes leading to greater efficiency. It is noteworthy that the difference between the D1 and D2 distributions is considerably smaller than the difference to the D3 distribution. A large fraction of this difference will be due to the increase in grain size, however it was expected that the increased spread seen in Fig. 9 would partially offset this decreased efficiency, as is the case between D1 and D2. This increased spread is particularly useful at increased inclinations as it spreads the insolation change away from being a very narrow band to a slightly wider band.

An additional factor must now be taken into account to correct for black body radiation and reflection of sunlight, as described in Pearson et al. (2006). These factors will lead to a reduced efficiency of the ring over the day side of the Earth, primarily due to thermal radiation from the particles, and an increased temperature over the night side of the Earth due to reflection of sunlight. Individually the dust particles have a small effect, however, due to the large number of particles used these two effects combined can reduce the effectiveness of the ring by approximately $40 \%$. This gives an estimate of the final mass of the ring of $1 \times 10^{12} \mathrm{~kg}$ for the lowest mass case. This mass is still smaller than the value of $2.3 \times 10^{12} \mathrm{~kg}$ seen in Pearson et al. (2006). The fact that the particles used by Pearson have a mean radius of $2.35 \mu \mathrm{m}$ would suggest that the method used in this paper should have a larger mass. This is not the case however, as the mass of a grain size distribution is dominated by larger dust grain sizes, which range up to $24.5 \mu \mathrm{m}$ in Pearson whereas in this paper the most pessimistic distribution, D3, only just reaches this upper limit.

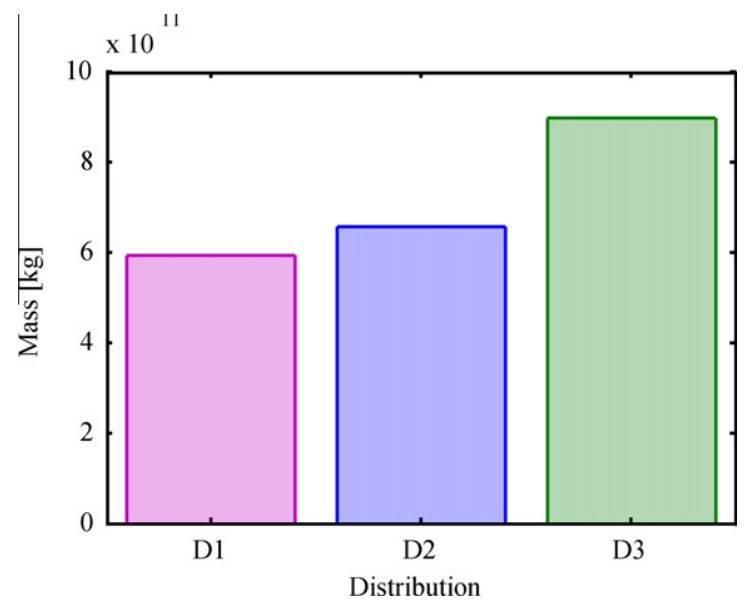

Fig. 14. Mass required to give an average $1.7 \%$ insolation change for three dust distributions. 
The orbital dynamics used in this paper demonstrate, as can be seen in Fig. 2, that the smallest particle sizes used in Pearson et al. (2006) would quickly deorbit due to atmospheric drag. The inclusion of the effects of solar radiation pressure and the $J_{2}$ effect and their use to find heliotropic ring patterns that will be long-lived will also account for a reduction in the overall mass in comparison to Pearson. In addition, this is essential for the success of such a proposal as the one presented here as the length of time that such a scheme must be in place cannot be known and would depend on many factors related to tackling global warming.

The insolation change as a function of time can be seen in Fig. 15. It shows that the insolation change is variable with a wide, flat peak greater than the $1.7 \%$ required but depressions as low as $0.5 \%$. These depressions occur during spring and autumn where the inclination of the equatorial to ecliptic plane is low. During these phases the view of the ring, as seen in Fig. 7 , is reduced to $2 \delta i R_{\max }$ at its maximum.

Also of interest is an understanding of where the insolation change over the Earth's surface will be greatest. An analysis of this can be seen in Fig. 16. Clearly the greatest effect will be felt in the equatorial and low tropical regions. In addition, it can be seen in Fig. 7 that each hemisphere experiences the greatest effects during its winter season. The precise effect that this will have on the local climate requires high-fidelity climate simulation. The amount of sunlight reflected onto the night side of the Earth can be estimated as well as the infra-red radiation received from the ring. Assuming no attenuation of thermal and visible radiation through the ring, an average power of $5 \times 10^{14} \mathrm{~W}$ and $3 \times 10^{14} \mathrm{~W}$ will be received by the night side of the Earth in the infra-red and visible spectrums respectively. Averaged over the cross-sectional area of the Earth gives an incident solar flux of $2.3 \mathrm{~W} \mathrm{~m}^{-2}$, a value that is several orders of magnitude greater than the $0.0017 \mathrm{~W} \mathrm{~m}^{-2}$ of sunlight reflected onto Earth by the Moon. The averaged flux in the infra-red regime is

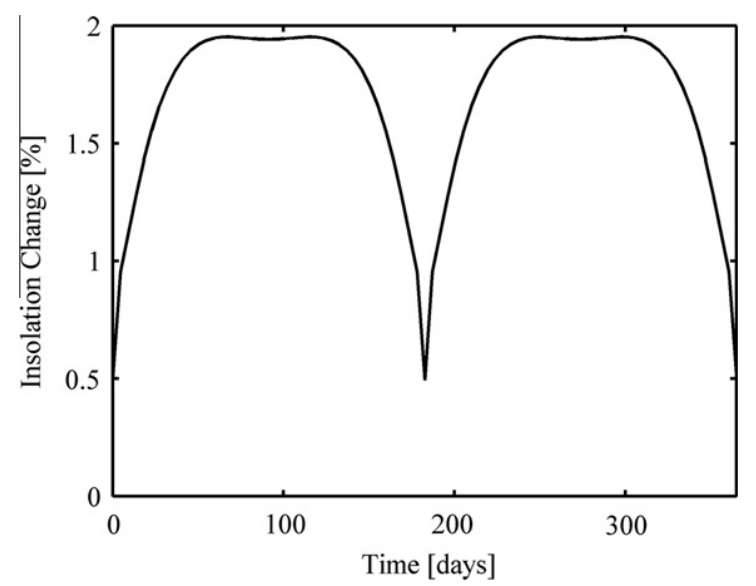

Fig. 15. Variation in insolation over the course of a year, giving an average of $1.7 \%$.

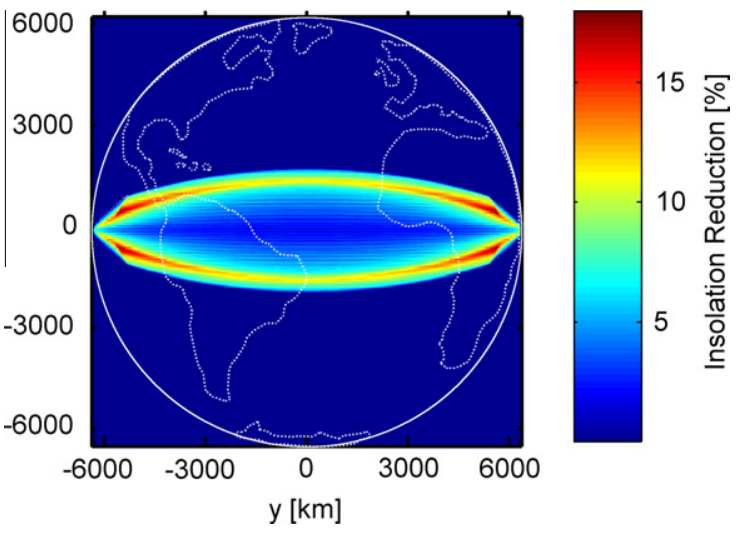

Fig. 16. Insolation change over the Earth's surface over the course of a year, as seen from the Sun.

$4 \mathrm{~W} \mathrm{~m}^{-2}$. Naturally this will have a significant effect on astronomy on Earth and will necessitate an increased number of space telescopes.

There are other effects that this form of geoengineering scheme will have on the Earth, most notably there will be a danger to spacecraft. The ring will generally inhabit an orbital region above LEO and below the high MEO orbits of the satellite navigation constellations. However, spacecraft in equatorial geostationary transfer orbits (GTO) will pass through the ring and would need protective shielding for this passage. It can be estimated that a spacecraft $2 \mathrm{~m}$ in radius will collide with a maximum of $4 \times 10^{14}$ particles, a figure which is dominated by a small portion of the ring, as seen in Fig. 12. However, higher inclination GTOs can be used to avoid the high density regions to greatly reduce the shielding requirement. Also a small fraction, $<1 \%$, of very fine grained material will de-orbit shortly after injection. These grains have radii of less than 6 microns. They will pass through the busiest region of LEO $(<500 \mathrm{~km})$ quickly because of their very high area-to-mass ratio and thus pose little danger to satellites there. The long lived nature of the greatest part of the rings particles leads to the disadvantage that it cannot easily be removed. To reduce these risks the ring should be populated over many years to enable close monitoring of the effect on the climate.

Consideration must be taken over the implementation of the Earth ring scenario. Though the principles of a change in the incoming solar flux over the Earth, and the climatic changes that occur, are reasonably well understood, the large zonal differences in insolation reduction has no historical data with which the climate modelling of the scenario can be correlated. Therefore the Earth ring concept must be implemented with great care. Firstly, the ring must be populated slowly over many years, over many phases, ensuring at each step the effect that the insolation reduction has on the Earth climate system is observed. It is likely that this implementation will occur in stages, with a period of implementation where dust is injected into the ring followed by a period of observation. The observation period could be a variety of lengths depending on the time 
available and the level of risk that is deemed acceptable. For example the relaxation time for the atmosphere is a matter of weeks whilst for the upper ocean it is several years, when considering the deep ocean this period is much greater. It is most prudent to observe for the full relaxation period of the upper ocean, however, this will greatly reduce the timeliness of this method of geoengineering and therefore, if the need arises, it can be imagined that the observation time will be less than this.

Ultimately the insertion of the ring around Earth will likely take many decades, unless a situation is reached where a climate fix is urgently required in which case drastic and highly risky measures may appear attractive, particularly ones which, once implemented, promise long periods of cooling, such as for this Earth ring method. However, some thought must be taken over the deactivation of the Earth ring concept. Since it is designed, or intended, to remain in position for many decades or even centuries a time may come when the ring is no longer required, or any side effects arising become too unpalatable for the cooling level achieved. In this situation the ring must be disposed of. The precise method by which this can be achieved has not been studied in detail as it is assumed that the ring, if ever implemented will be used over a long timeframe, at the end of which technology will have advanced greatly and will thus make the disposable of the ring a more simple task. A few thoughts can be expressed on possible disposable mechanisms however. Firstly, there are ongoing investigations into debris removal mechanisms for low Earth orbit, such as laser ablation of debris objects or sweeping out orbits with a giant "catchers mitt", and it is assumed that, should these methods be used in LEO, they can be used to 'tidy up' the Earth ring. Secondly, it can be observed in Saturn's ring system that there are many gaps within the rings. These are regions swept out by Saturn's many moons. It is assumed therefore that the same principle can be applied to the Earth ring. A sufficiently large asteroid can be captured into the required orbit to sweep up the dust ring material or alter the dust grains orbits by gravitational attraction such that they decay or escape the Earth system. This process will take many years to complete.

Another side effect that must be discussed is the attenuation of communication signals from geostationary satellites. It is important to consider this since the majority of telecommunications utilises satellites in the geostationary ring. By taking the maximum extent of the Earth ring out of the equatorial plane the maximum latitude where a telecommunication signal can be attenuated is determined to be $0.45^{\circ}$. This is a small range of latitudes that does not encompass any of the world's major cities.

The approximate attenuation can be determined using the assumption that the interaction between the asteroid dust grains and the microwave signal is the same as for sand grains, a phenomenon which has been studied by Hong et al. (2008). These results show that, at a frequency of $36.5 \mathrm{GHz}$, the minimum extinction efficiency unit is 0.1 for a size parameter of approximately 0.5 . This size parameter equates to a grain radius of $650 \mu \mathrm{m}$ which is much greater than the grains required by the dust ring. Since the results from (Hong et al., 2008) show that below this size parameter the extinction efficiency reduces an upper estimate of the attenuation can be made using $Q_{\text {ext }}=0.1$. Since $Q_{\mathrm{ext}}=\sigma_{\mathrm{ext}} / \pi R^{2}$ the extinction cross section, $\sigma_{\mathrm{ext}}$, is estimated to be $1.3 \times 10^{-10} \mathrm{~m}^{2}$ For the attenuation calculation the Beer-Lambert law is used with the assumption of a homogeneous grain with the maximum path length through the ring used to provide the upper estimate of the attenuation. This gives a maximum attenuation of 0.5 though the majority of path through the ring will give an attenuation much less than this. For the regions covered by the ring this reduction in communications signal strength will be a concern though it can be imagined that ground based communications links can be established from an unaffected region should this attenuation become problematic.

\section{Conclusions}

The concept of an Earth ring comprised of dust grains to offset global warming, first investigated in (Pearson et al., 2006), has been investigated by considering novel families of heliotropic orbits. By the inclusion of the perturbations of solar radiation pressure and the $J_{2}$ effect the orbital dynamics of these high area-to-mass ratio dust grains has been analysed in the $e-\phi$ phase space using a Hamiltonian approach. From this analysis stable, sun-pointing orbits have been found that enable a ring to remain in equilibrium for long periods. Using these orbits as a location for geoengineering enables an estimate of the mass of dust required to offset a $2{ }^{\circ} \mathrm{C}$ increase in temperature increase by a $1.7 \%$ reduction in solar insolation. The result of this estimate is a dust mass of $1 \times 10^{12} \mathrm{~kg}$, including a reduced efficiency due to particle emission and reflection, which is less than the dust cloud methods found in Pearson et al. (2006) and Struck (2007) but larger than the solid reflector method suggested in McInnes (2010) and the refractor method seen in Angel (2006). The use of dust rather than large reflective or refractive devices has the great advantage of reducing the complexity of the system and also, if the dust is sourced from an asteroid, reduces greatly the launch costs of the system. However, despite these improvements over other space-based geoengineering schemes, the unresolved engineering aspects, of the Earth ring concept, such as the mechanism for capture of an appropriate asteroid, mean that it can only be viewed as a far term method for combating climate change. Future work will use a more accurate model to take into account the complex three-dimensional structure of the ring.

\section{Acknowledgement}

This work was funded by the European Research Council grant 227571 (VISIONSPACE). 


\section{Appendix A. Supplementary data}

Supplementary data associated with this article can be found, in the online version, at http://dx.doi.org/10.1016/ j.asr.2012.10.024.

\section{References}

Angel, R. Feasibility of cooling the Earth with a cloud of small spacecraft near the inner Lagrange point (L1). PNAS 103, 17184-17189, 2006.

Bewick, R., Sanchez, J., McInnes, C.R., An L1 positioned dust cloud as an effective method of space-based geo-engineering. in: International Astronautical Congress. Prague: IAC-10.D11.11.17, 2010.

Bewick, R., Sanchez, J.P., McInnes, C.R. The feasibility of using an L1 positioned dust cloud as a method of space-based geoengineering. Adv. Space Res. 49, 1212-1228, 2012.

Colombo, C., McInnes, C.R. Orbital dynamics of 'smart dust' devices with solar radiation pressure and drag. J. Guid. Control Dyn. 34, 1613-1631, 2011.

Colombo, C., Lücking, C., McInnes, C.R. Orbital dynamics of high areato-mass ratio spacecraft with $\mathrm{J} 2$ and solar radiation pressure for novel earth observation and communication services. Acta Astronautica 81, $137-150,2012$

Govindasamy, B., Caldeira, K. Geoengineering Earth's radiation balance to mitigate $\mathrm{CO}_{2}$-induced climate change. Geophys. Res. Lett. 27, $2141-$ 2144,2000

Govindasamy, B., Caldeira, K., Duffy, P.B. Geoengineering Earth's radiation balance to mitigate climate change from a quadrupling of $\mathrm{CO}_{2}$. Global Planet. Change 37, 157-168, 2003.
Hamilton, D.P., Krivov, A.V. Circumplanetary dust dynamics: effects of solar gravity, radiation pressure, planetary oblateness, and electromagnetism. Icarus 123, 503-523, 1996.

Hong, G., Yang, P., Weng, F., et al. Microwave scattering properties of sand particles: Application to the simulation of microwave radiances over sandstorms. J. Quant. Spectrosc. Rad. 109, 684-702, 2008.

IPCC, Contribution of working groups I, II and III to the fourth assessment report of the intergovernmental panel on climate change, Core Writing Team, in: Pachauri, R.K. and Reisinger, A. (Eds.), IPCC, Geneva, Switzerland. pp 104, 2007.

Krivov, A.V., Getino, J. Orbital evolution of high-altitude balloon satellites. Astron. Astrophys. 318, 308-314, 1997.

Krivov, A.V., Sokolov, L.L., Dikarev, V.V. Dynamics of Mars-orbiting dust: Effects of light pressure and planetary oblateness. Celest. Mech. Dyn. Astr. 63, 313-339, 1995.

McInnes, C.R. Space-based geoengineering: challenges and requirements. P. I. Mech. Eng. C-J Mech. 224, 571-580, 2010.

Pearson, J., Oldson, J., Levin, E. Earth rings for planetary environment control. Acta Astronaut. 58, 44-57, 2006.

Sanchez, J.P., McInnes, C. Asteroid resource map for near-Earth space. J. Spacecraft Rockets 48, 153-165, 2011.

Seifritz, W. Mirrors to halt global warming. Nature 340, 603, 1989.

Shepherd, J., Caldeira, K., Cox, P. et al., Geoengineering the climate. in: Report of Royal Society Working Group of Geo-engineering, 2009.

Struck, C. The feasibility of shading the greenhouse with dust clouds at the stable lunar Lagrange points. JBIS 60, 82-89, 2007.

Wilck, M., Mann, I. Radiation pressure forces on "typical" interplanetary dust grains. Planet. Space Sci. 44, 493-499, 1996. 